\title{
The impact of activating source dwell positions outside the CTV on the dose to treated normal tissue volumes in TRUS guided 3D conformal interstitial HDR brachytherapy of prostate cancer
}

\author{
Leif Karlsson, BSc',2, Per Thunberg, PhD',2, Bengt Johansson, PhD³, Jan Persliden, PhD 1,2 \\ 'Department of Medical Physics, Örebro University Hospital, Örebro, Sweden, ${ }^{2}$ School of Health and Medical Sciences, Örebro University. \\ Örebro, Sweden, ${ }^{3}$ Department of Oncology, Örebro University Hospital, Örebro, Sweden
}

\begin{abstract}
Purpose: Dose coverage is crucial for successful treatment in mono-brachytherapy. Since few and very high dose fractions are used, there is an important balance between dwell positioning outside the clinical target volume (CTV) and possible damage on adjacent normal tissue. The purpose of this study was to evaluate the possibility of having dwell positions close to the CTV surface, while maintaining an acceptable dose distribution, and to investigate the robustness in terms of known geometrical uncertainties of the implant.

Material and methods: This study included 37 patients who had received brachytherapy for prostate cancer as a monotherapy with the following schedules: $2 \times 14$ Gy or $3 \times 11 \mathrm{~Gy}$, each fraction separated by two weeks. The source dwell positions were activated $5 \mathrm{~mm}$ outside CTV. New optimizations were simulated for dwell positions at 3, 2, 1, and $0 \mathrm{~mm}$. Inverse and graphical optimization were applied according to the relative dose constraints: $\mathrm{V}_{100 \mathrm{CTV}} \geq 97 \%$,

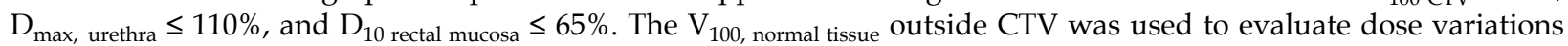
caused by different dwell positions. Prostate geometries and dose distributions for the different dwell positions outside the CTV were used to investigate the impact on the CTV dose distribution due to geometrical uncertainties.

Results: Both $\mathrm{V}_{100, \mathrm{CTV}}$, and $\mathrm{V}_{100 \text {, normal tissue }}$ decreased, $98.6 \%$ to $92.2 \%$, and $17 \mathrm{~cm}^{3}$ to $9.0 \mathrm{~cm}^{3}$, for dwell activation from $5 \mathrm{~mm}$ to $0 \mathrm{~mm}$. The evaluation of both simulated longitudinal geometrical uncertainties and different source dwell activations implied that $\mathrm{V}_{100 \text {, CTV }}$ ranged from $98.6 \%$ to $86.3 \%$.

Conclusions: It is possible to reduce the $\mathrm{V}_{100 \text {, normal tissue }}$ by decreasing the source dwell positions outside the CTV from 5 to $3 \mathrm{~mm}$, while maintaining dose constraints. In combination with the estimated geometrical uncertainties, however, the source dwell positions need to be $5 \mathrm{~mm}$ from the surface in order to maintain a robust implant.
\end{abstract}

Key words: brachytherapy, margins, optimization, prostate cancer.

\section{Purpose}

In the latter part of the 1990s, an alternative treatment for prostate cancer emerged where interstitial high dose rate (HDR) brachytherapy was applied as a boost in combination with external beam radiation therapy (EBRT) [1-5]. This brachytherapy treatment technique is also used for mono-therapy of prostate cancer for multiple fractions per implant or one fraction per implant [6-9]. Since 2004, the latter is the standard treatment at our hospital. The treatment technique has gradually been developed including major advances, such as the use of appropriate treatment planning systems [10-12]. The introduction of the new treatment planning system Oncentra Prostate ${ }^{\circledR}$
(Elekta AB, Stockholm, Sweden), gave the possibility, in combination with trans-rectal ultrasound (TRUS) to in real time optimise the needle configuration and the dose distribution in an optimal 3D conformal way during the procedure. All necessary corrections of needle displacement, movements, and/or deformation of clinical target volume (CTV) and organs at risk (OARs) after placement of the needles in the fixed treatment geometry, can be performed for each fraction if one fraction per implant is used. Interstitial brachytherapy is an old radiation treatment technique [13] based on an extended implanted volume of the CTV in order to reach optimal dose coverage. Even with the modern techniques of stepping source and 
dose optimization, there is a need for some over-implantation, especially with an ellipsoidal shaped volume like the prostate gland. At our department, the standardized activation of source dwell positions, including over-implantation, are localized $5 \mathrm{~mm}$ outside the CTV surface. A possible consequence of the over-implantation is radiation damage to normal tissue close to the prostate gland. A perfect dose coverage is crucial in mono-brachytherapy treatment, where only 1-3 fractions with very high dose are more commonly used. This is due to the low $\alpha / \beta$ ratio of prostate cancer cells. Therefore, there is an important balance in interstitial HDR brachytherapy treatment of prostate regarding the over-implantation by the active dwell positions outside CTV, and the possible damage on the adjacent normal tissue.

The aims of this study were: 1) to evaluate the possibility of activating dwell positions closer to the CTV surface, while maintaining the same dose distribution based on our dose constraints, and thereby minimise the extent of treating normal tissue around the CTV; 2) to investigate the robustness of the dose distribution, in terms of known geometrical uncertainties.

\section{Material and methods}

\section{Patients}

Thirty-seven patients were included into this retrospective study, who had received brachytherapy treatment for prostate cancer as a mono-therapy during 20102011. They had been treated according to two different treatment schedules: $2 \times 14$ Gy and $3 \times 11$ Gy. Each fraction, separated by two weeks, given as a single implant. Only one fraction from each patient was used in the simulations. The main setting-up of the first dwell position was $5 \mathrm{~mm}$ from the base-plane. Moreover, the relative dose constraint $\mathrm{V}_{100}$ for CTV as a volume, which is defined by 100 percentage of the prescribed dose was: $V_{100, \text { СTV }} \geq 97 \%$. The dose constraints for normal tissue, as a percentage of the prescribed reference dose, for both treatment schedules were: $D_{\text {max, urethra }} \leq 110 \%$ and $D_{10}$ rectal mucosa $\leq 65 \%$. The first 37 patients fulfilling these criteria were included in the analyses.

This study was approved by the local Ethic Committee (DNR 2012/177).

\section{Treatment technique}

The treatment technique involved a stepper unit with a needle template TRUS (Pro Focus, BK Medical, Herlev, Denmark) and a treatment planning system Oncentra Prostate ${ }^{\circledR}$ version 3.2.2/4.0.7, OCP (Elekta AB, Stockholm, Sweden) which was later integrated with ultrasound PROSIUS ${ }^{\circledR}$ (Elekta AB, Stockholm, Sweden). Between 15 and 20 stainless needles were used depending on the prostate volume. The implantation was performed under spinal anaesthesia. A Foley catheter was inserted into the bladder and an X-ray marker was placed into the catheter. The prostate gland was localized with TRUS and aligned with the template holes. Two anchor needles were placed into the prostate to prevent or minimise the longitudinal movement of the prostate gland.
A series of transversal US-images with a $1 \mathrm{~mm}$ slice thickness were acquired and converted to a 3D-volume. A base plan was defined at the base of the prostate, as a longitudinal reference. The CTV (prostate capsule without a margin) and urethra were outlined, and a simulated needle configuration was manually created, referred to as the pre-plan in OCP. The needles were virtually placed in the shape of two rings. One inside the prostate gland 3-5 mm from the surface of the largest CTV-structure and the other was set to cover the apex and the base plane (Fig. 1). The needles were then inserted to the prostate according to the pre-plan. The first needle was placed with the tip positioned $25 \mathrm{~mm}$ above the pre-base plane (the $1^{\text {st }}$ dwell position is possible $10 \mathrm{~mm}$ from the needle top). This takes into account, from our experience, a longitudinal movement of the prostate gland. Moreover, it allows to activate the first dwell position $5 \mathrm{~mm}$ above the base plane. After the determination of the insertion length of the first needle, the other needles were prepared to the same length.

If the prostate displacement was more than $10 \mathrm{~mm}$, a correction was made of the needle length by pushing in all the needles an additional $10 \mathrm{~mm}$. Sometimes it was not possible to achieve this, and the first dwell position will end up closer than $5 \mathrm{~mm}$ from the base which can result in an under dosage of the CTV. During the needle insertion, a needle position was corrected if necessary. A needle can be added depending on the situation, e.g. interference from bones or due to real time circumstances.

After the needles were placed, a new baseplane was defined, reflecting the real longitudinal displacement of prostate gland and a new US-scan (live scan) was generated with the needles in place, defining the treatment geometry. The US-probe was placed at the base plane and two orthogonal X-ray images were taken. In this reconstructed volume, new structures for CTV and OARs were outlined. Corrections were made for the longitudinal and radial displacement of the needles, compared to the preplan, by reconstructing the needles. The position of the needle tip in relation to the base plane was determined from the geometry, the lateral X-ray image, and the US scans in order to minimize the geometrical uncertainties in the longitudinal direction. Dwell positions were virtually activated in the needles, up to $5 \mathrm{~mm}$ outside the CTV. Finally, a dose distribution was obtained, based on

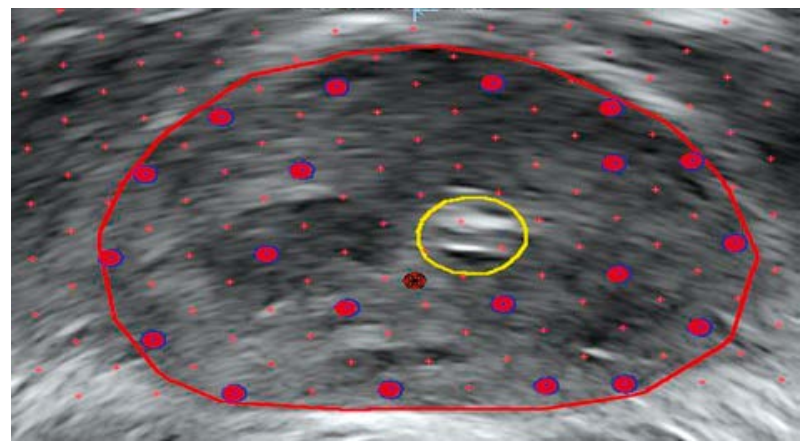

Fig. 1. An example of a virtual needle positioning, formed as a ring just inside CTV (red solid line) and one ring around urethra (yellow) 
inverse and graphical optimization, according to local dose constraints. The evaluation was based on dose volume histogram (DVH) parameters. During the analyses, only relative dose values were used.

\section{The influence of dwell positioning outside the CTV (Analysis 1)}

New dose optimizations were simulated for the given treatment geometry for different source dwell activations outside the CTV: $3 \mathrm{~mm}, 2 \mathrm{~mm}, 1 \mathrm{~mm}$, and $0 \mathrm{~mm}$. The dwell positions were activated according to the rules in the treatment planning system [14] (Fig. 2). The same routines as for the clinical procedure were used when performing these simulations. Inverse optimization, followed by graphical optimization were used, trying to reach the relative dose constraints with the following priority (a-c): (a) $\mathrm{D}_{\text {max, urethra }} \leq 110 \%$, (b) $\mathrm{D}_{10}$ rectal mucosa $\leq 65 \%$, (c) $V_{100, \text { CTV }} \geq 97 \%$. In order to evaluate the variations in the treated normal tissue volume outside the CTV, a new volume (called large) encompassing the $100 \%$-isodose was

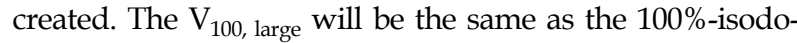
se volume. The normal tissue volume with a dose higher or equal to $100 \%$ can be calculated according to the following expression.
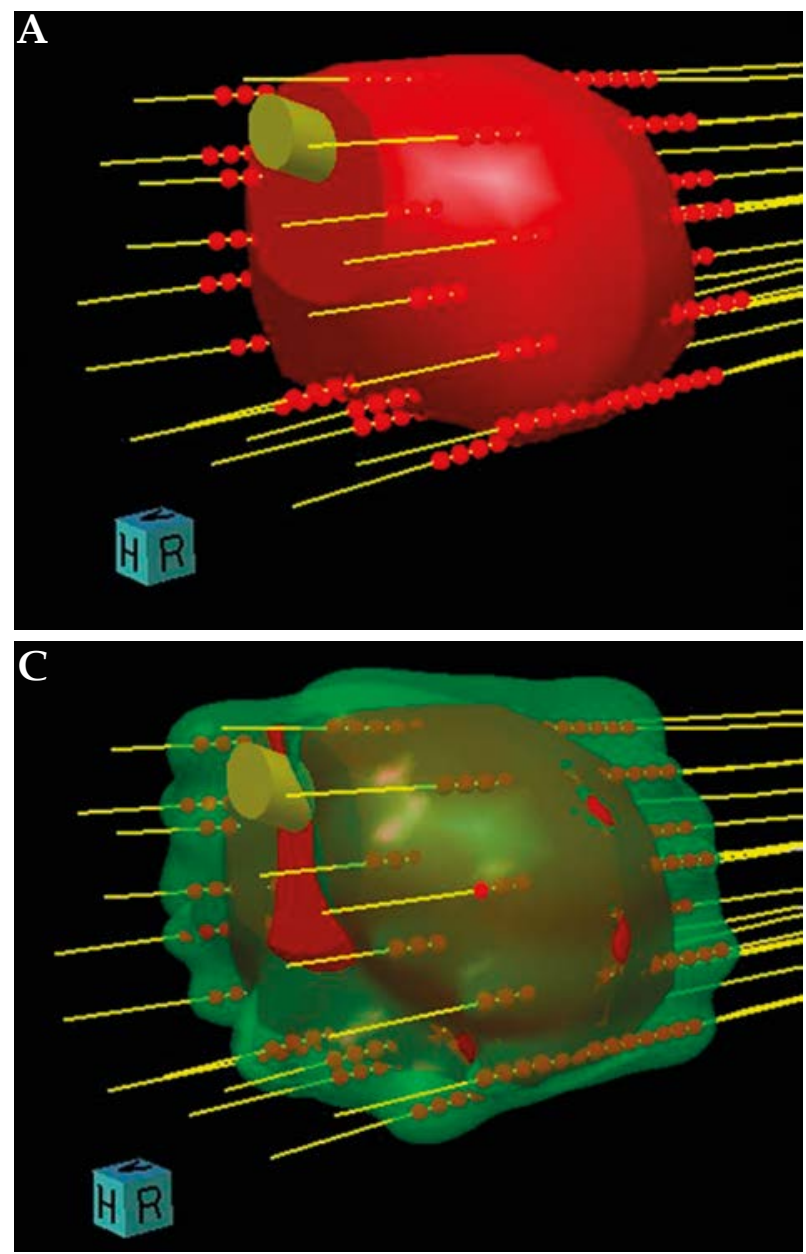

Fig. 2. An example of needle configuration with dwell activation with and without $100 \%$-isodose, $5 \mathrm{~mm}$ outside CTV surface

$(A, C)$, and on the surface $(B, D)$. The red surfaces show the outlined prostate $(C T V)$ and the green surfaces illustrates the volumes where the dose constraint $V_{100}$ were fulfilled

$\mathrm{V}_{100, \text { normal tissue }}=\mathrm{V}_{100, \text { large }}-\mathrm{V}_{100, \mathrm{CTV}}-\mathrm{V}_{100, \text { urethra }}$

Dose volume histogram data was used to compare the simulated dose distributions with the original dose distribution. The following DVH parameters were determined and described: CTV: $\mathrm{V}_{100}, \mathrm{~V}_{150}, \mathrm{~V}_{200}, \mathrm{D}_{98}, \mathrm{D}_{90}$; urethra: $\mathrm{D}_{\text {max }}, \mathrm{D}_{10}$; rectal mucosa: $\mathrm{D}_{10}$; conformal index (COIN) [15]. COIN-index reflects the relation between the dose coverage of the CTV and the amount of irradiated normal tissue outside the CTV. The urethra volume inside CTV is excluded in the DVH-calculations.

\section{The influence of geometrical uncertainties on the dose distribution (Analysis 2)}

Geometrical uncertainties in this treatment technique are mainly connected with the longitudinal direction of the needles. These uncertainties include the reconstruction of the first dwell position, i.e. the needle tip $( \pm 1.5 \mathrm{~mm})$, mechanical positioning of the source in the needle $( \pm 1 \mathrm{~mm})$, and the length of a stainless needle $( \pm 1 \mathrm{~mm})$. The intra-fraction movements of needles and prostate gland in this study was estimated to be in order of $1 \mathrm{~mm}$, based on the work of Milickovic et al. [16]. Calculating an absolute error of these uncertainties will give
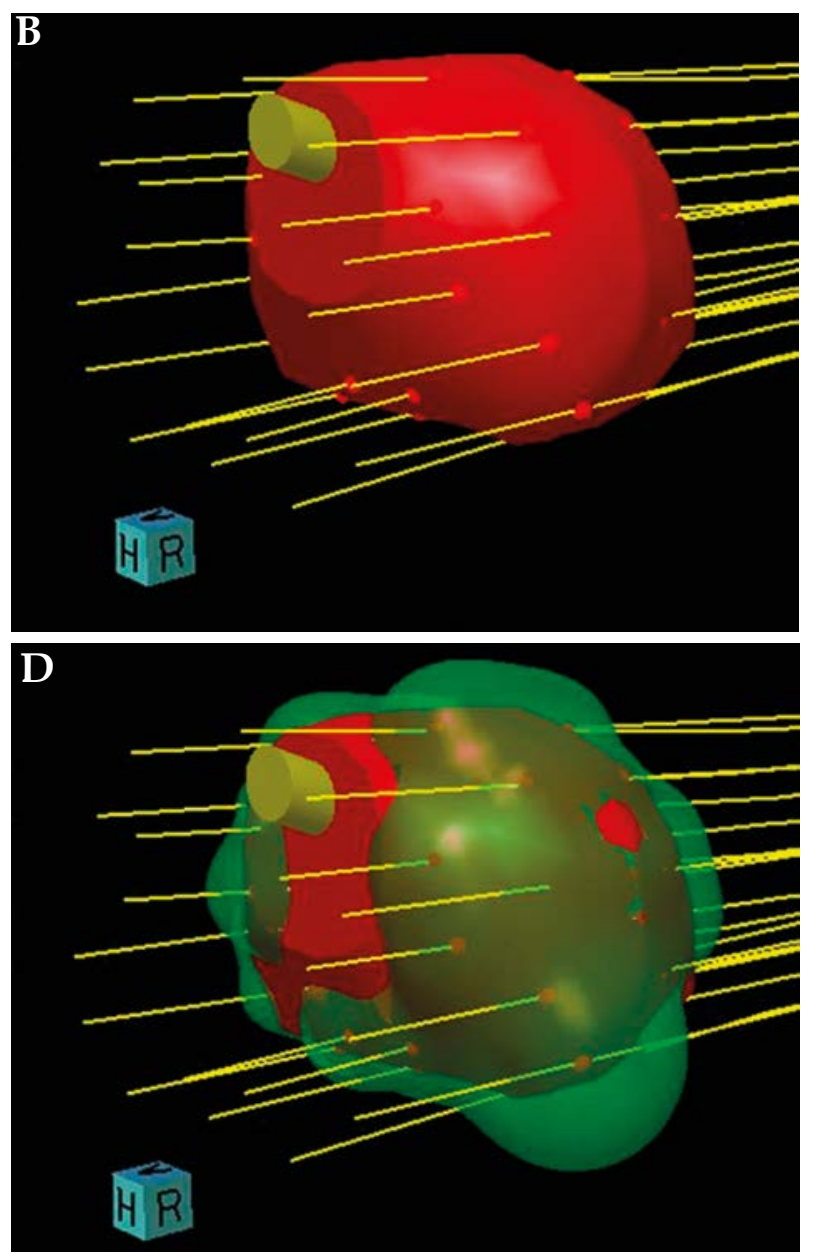

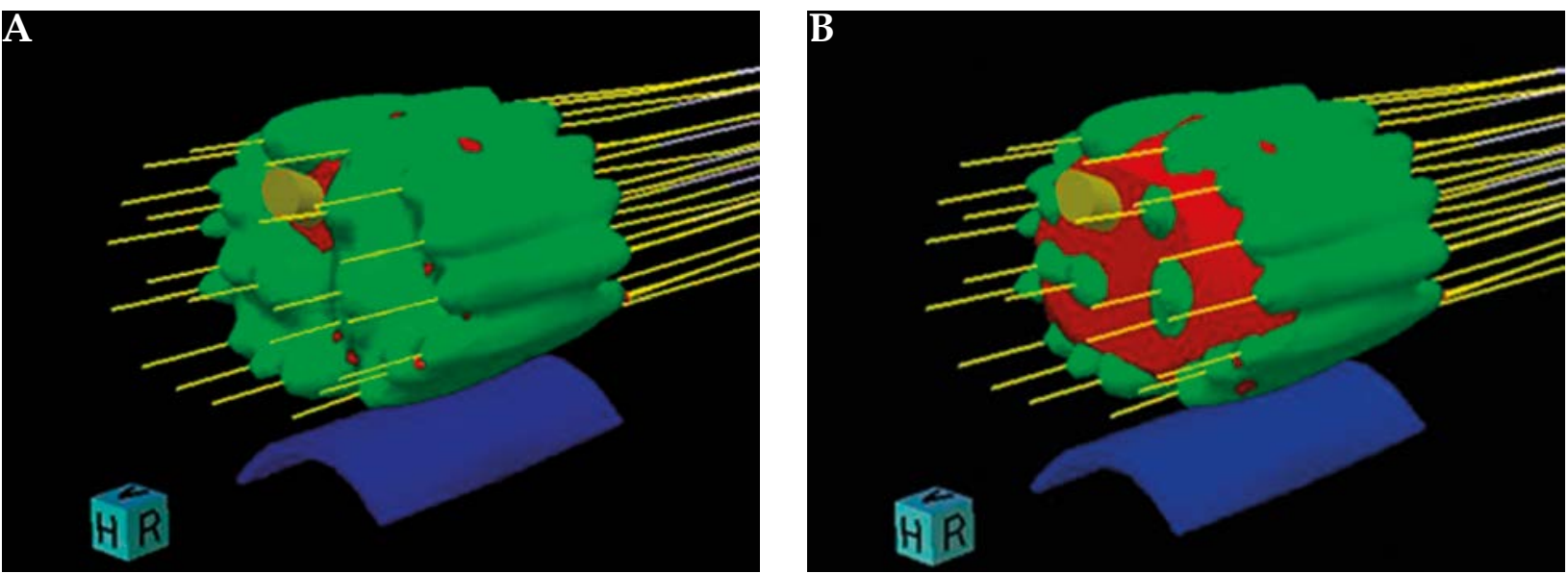

Fig. 3. A 3D-image showing a visual relationship between CTV (red) and the reference dose (green) for: (A) no simulated needle displacement and (B) a simulated displacement of $4 \mathrm{~mm}$. Blue region shows the position of the rectal mucosa

an estimated total longitudinal geometrical uncertainty of $\pm 2.3 \mathrm{~mm}$. There is also an uncertainty of approximately $0.5 \mathrm{~mm}$ in the radial direction from the needle, which is not included in the analyses of this study.

In Analysis 1, dwell positions were placed closer to the CTV surface for the simulated dose distributions then for the real one. This will probably cause a dose distribution, which is more sensitive to organ and needle movements, i.e. less robust. The patient geometries and their simulated dose distributions were used to simulate longitudinal displacements of the needles by retracting the needles and the dose distribution in relation to the CTV and OAR:s (Fig. 3). For each displacement new DVH-data was calculated. The simulated displacements of $1 \mathrm{~mm}$, $2 \mathrm{~mm}, 3 \mathrm{~mm}$, and $4 \mathrm{~mm}$ were calculated in order to estimate the uncertainties. An estimation of extent to which the robustness of the dose distribution will be changed by using DVH-data was performed.

\section{Statistics}

The data from each group, with different dwell positioning in Analysis 1 and for the simulated geometrical uncertainties in Analysis 2 was tested for normality using the Shapiro-Wilk test. All groups were then checked for significance $(p<0.05)$, both with paired students $t$-test if the data had a normal distribution (parametrical test) and Wilcoxon signed-ranks test (non-parametrical test) for data not having a normal distribution. The SPSS software was used for the statistical analyses.

\section{Results}

The matrix in Figure 4 summarize the results in terms of fulfilling the dose constraint $V_{100, \text { CTV }} \geq 97 \%$ for the different dwell activation positions and longitudinal displacements. In the case of no longitudinal displacement, the dose constraint $\mathrm{V}_{100, \mathrm{CTV}} \geq 97 \%$ was fulfilled for 30 patients with a dwell activation $3 \mathrm{~mm}$ from the CTV surface, 20 patients for $2 \mathrm{~mm}, 2$ patients for $1 \mathrm{~mm}$, and none for $0 \mathrm{~mm}$. As the longitudinal needle displacement increased from $1 \mathrm{~mm}$ to $4 \mathrm{~mm}$, the original dose distribution decreased for all dwell positions outside the CTV. For the dwell position of $5 \mathrm{~mm}$ outside the CTV, the $\mathrm{V}_{100 \text {, CTV }}$ decreased significantly from $98.6 \%$ (no displacement) to $95.4 \%$ for a displacement of $4 \mathrm{~mm}$. For a displacement of $3 \mathrm{~mm}$, the mean value of $\mathrm{V}_{100}$, CTV also decreased to a value lower than the dose constraint of $97 \%$, but the difference was not significant.

Figure 5 shows an individual example of DVH curves from the different simulations. The dashed curves shows how the DVH changes compared to the original DVH (solid line) when the most distal dwell positions coincides with the surface of CTV (Fig. 5B), the longitudinal displacement is $4 \mathrm{~mm}$ (Fig. 5C) or these two combined (Fig. 5D).

The dose volume parameters obtained from the simulations are presented in Table 1 . The dose constraints, $D_{\max \text {, urethra }} \leq 110 \%$, and $D_{10}$ rectal mucosa $\leq 65 \%$ were fulfilled in almost all the simulations. The mean values for the different dwell activation were for $D_{\max }$ urethra and

\begin{tabular}{|c|c|c|c|c|c|}
\hline \multirow{2}{*}{$\begin{array}{c}\text { Displacement } \\
{[\mathrm{mm}]}\end{array}$} & \multicolumn{5}{|c|}{ Distance to dwell position outside CTV [mm] } \\
\cline { 2 - 6 } & 5 & 3 & 2 & 1 & 0 \\
\hline 0 & 98.6 & 97.7 & $96.6^{\star}$ & 94.7 & 92.2 \\
& $37(37)$ & $30(37)$ & $20(37)$ & $2(37)$ & $0(37)$ \\
\hline 1 & 98.4 & 97.5 & 96.5 & 94.4 & 91.9 \\
& $37(37)$ & $28(37)$ & $13(37)$ & $2(37)$ & $0(37)$ \\
\hline 2 & 97.9 & $96.8^{*}$ & 95.7 & 93.4 & 90.7 \\
& $36(37)$ & $17(37)$ & $12(37)$ & $0(37)$ & $0(37)$ \\
\hline 3 & $96.9^{\star}$ & 95.6 & 94.3 & 91.6 & 88.8 \\
& $22(37)$ & $1(37)$ & $1(37)$ & $0(37)$ & $0(37)$ \\
\hline 4 & 95.4 & 93.8 & 92.2 & 89.1 & 86.3 \\
& $0(37)$ & $0(37)$ & $0(37)$ & $0(37)$ & $0(37)$ \\
\hline
\end{tabular}

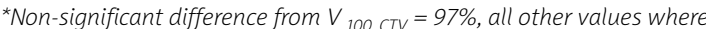
significant different ( $p$ value $<0.01$ ) from $V_{100, \text { cTV }}=97 \%$

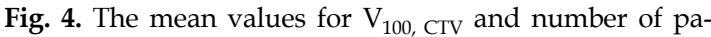
tients fulfilling $\mathrm{V}_{100, \text { CTV }}>97 \%$ for dwell activation at $3 \mathrm{~mm}$, $2 \mathrm{~mm}, 1 \mathrm{~mm}$, and $0 \mathrm{~mm}$ from the CTV surface in combination with the simulated needle displacement of $1 \mathrm{~mm}$ to $4 \mathrm{~mm}$ in the longitudinal direction. Green surfaces where $\mathrm{V}_{100, \text { CTV }} \geq 97 \%$ and red surfaces where $\mathrm{V}_{100, \text { CTV }} \leq 95 \%$ 

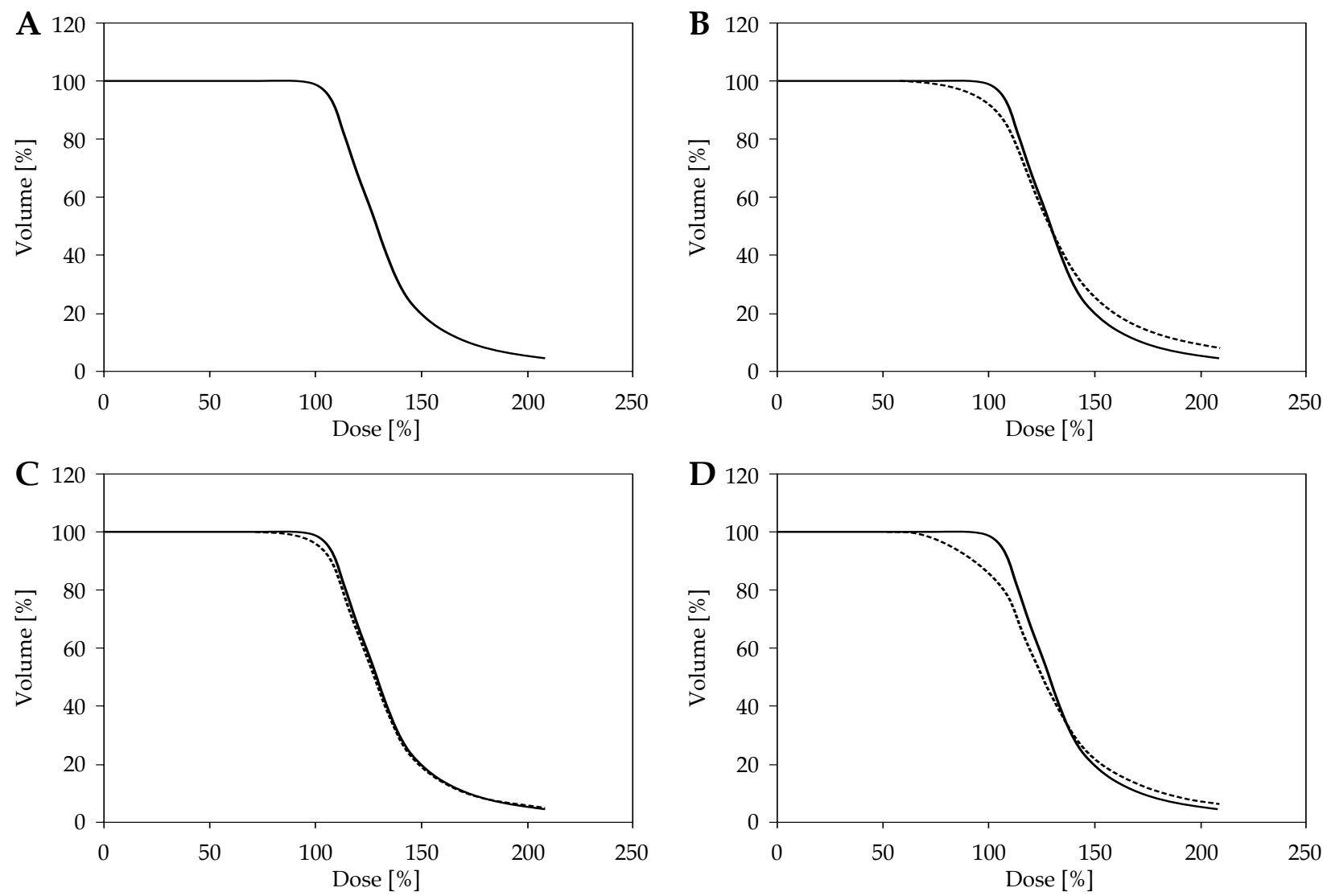

Fig. 5. DVH curves for CTV: (A-D) (- $\longrightarrow$ dwell positions placed $5 \mathrm{~mm}$ from the CTV surface, (B-D) (- - - - - -) in combination with the dwell positions placed on the CTV surface (B), simulated needle displacement of $4 \mathrm{~mm}$ in the longitudinal direction (C) with dwell positions placed on the CTV surface and simulated needle displacement of $4 \mathrm{~mm}$ in the longitudinal direction $(\mathrm{D})$

$\mathrm{D}_{10, \text { mucosa }} 109.9 \%$, and $56 \%$, respectively. The dose to rectal mucosa was almost not affected by the simulations as can be seen from the mean value.

The mean prostate volume for the patients included in this study was $36 \mathrm{~cm}^{3}$ (range: $21-58 \mathrm{~cm}^{3}$ ). The mean values of the normal tissue volume around the CTV given a dose of $100 \%$ or higher $\left(\mathrm{V}_{100, \text { normal tissue }}\right)$ for source dwell activations of 5 to $0 \mathrm{~mm}$ from the CTV surface were $17 \mathrm{~cm}^{3}$ to $9 \mathrm{~cm}^{3}$. This corresponds to $47-25 \%$ of the mean prostate gland volume. The results for $\mathrm{V}_{150, \text { normal tissue }}$ and $\mathrm{V}_{200 \text {, normal tissue }}$ for the same dwell activations varied from $3.0 \mathrm{~cm}^{3}$ to $1.7 \mathrm{~cm}^{3}$, and from $1.0 \mathrm{~cm}^{3}$ to $0.7 \mathrm{~cm}^{3}$. When comparing the dwell activation groups 5-3, 3-2, 2-1, 1-0, there were a significant difference between the groups for normal tissue, CTV, and COIN-index $(p<0.01)$, except for a few values indicated with an asterix $\left(^{*}\right)$ in Table 1.

\section{Discussion}

There are several decisions that have to be considered when treating prostate cancer with brachytherapy. Some of these considerations involve source dosimetry, treatment planning, and equipment performance [17]. This study has focused on geometrical uncertainties and the positioning of the dwells relative to the CTV, and the surrounding normal tissue. The results of Analysis 1 showed that it is possible to reduce the treated normal tissue around the prostate gland by reducing source dwell activations outside the prostate gland. The mean value

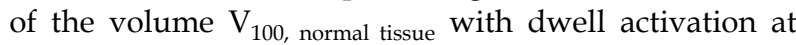
$5 \mathrm{~mm}$ from the prostate surface (clinical situation) was $17 \mathrm{~cm}^{3}$, whilst for $2-12 \mathrm{~cm}^{3}$ (simulation). If these volumes instead were related to an added radius on the prostate gland by approximating the prostate gland volume $\left(36 \mathrm{~cm}^{3}\right)$ to a sphere, the radius would be $3 \mathrm{~mm}$ and $2.5 \mathrm{~mm}$, respectively. This is in order of the estimated geometrical uncertainties.

The results also showed a decrease in $V_{150 \text {, normal tissue }}$

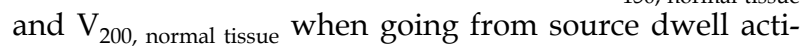
vation at $5 \mathrm{~mm}$ to dwell activation on the CTV surface, but $\mathrm{V}_{150, \mathrm{CTV}}$ and $\mathrm{V}_{200 \text {, CTV }}$ was increasing. This means that these volumes, $V_{150, \text { CTV }}$ and $V_{200, \text { CTV }}$ are gradually increasing within the prostate gland and the risk of interference with the urethra is increased. In recent review studies $[17,18]$, the uncertainties in brachytherapy has been investigated. For the treatment technique used in this study (TRUS guided 3D conformal interstitial brachytherapy HDR of prostate cancer), the geometrical uncertainty has been estimated to be in order of $1.5 \mathrm{~mm}$ [18]. Siebert et al. 
Table 1. Mean values $(\mathrm{m})$, standard deviation and range for dose volume parameters from simulated dose distributions for different distances of dwell positioning from CTV surface. The asterix ${ }^{*}$ ) indicates group comparisons where no statistical difference $(p<0.01)$ occurred

\begin{tabular}{|c|c|c|c|c|c|c|c|c|c|c|}
\hline \multirow{2}{*}{$\begin{array}{l}\text { Dwell } \\
\text { position }\end{array}$} & \multicolumn{2}{|c|}{$5 \mathrm{~mm}$} & \multicolumn{2}{|c|}{$3 \mathrm{~mm}$} & \multicolumn{2}{|c|}{$2 \mathrm{~mm}$} & \multicolumn{2}{|c|}{$1 \mathrm{~mm}$} & \multicolumn{2}{|c|}{$0 \mathrm{~mm}$} \\
\hline & $m \pm 2 \sigma$ & Range & $m \pm 2 \sigma$ & Range & $m \pm 2 \sigma$ & Range & $m \pm 2 \sigma$ & Range & $m \pm 2 \sigma$ & Range \\
\hline \multicolumn{11}{|l|}{ Normal tissue } \\
\hline $\begin{array}{l}V_{100, \text { normal tissue }} \\
{\left[\mathrm{cm}^{3}\right]}\end{array}$ & $17.0 \pm 5.4$ & $11.2-22.1$ & $14.0 \pm 4.2$ & $10.7-18.5$ & $12.0 \pm 4.4$ & $8.1-17.8$ & $10.0 \pm 4.6$ & $6.5-17.2$ & $9.0 \pm 4.0$ & 5.3-13.2 \\
\hline $\begin{array}{l}V_{150, \text { normal tissue }} \\
{\left[\mathrm{cm}^{3}\right]}\end{array}$ & $3.0 \pm 1.4$ & $1.6-4.5$ & $2.6 \pm 1.0$ & $1.5-3.9$ & $2.2 \pm 1.0$ & $1.5-3.5$ & $1.8 \pm 1.0^{\star}$ & $0.5-2.9$ & $1.7 \pm 1.0^{\star}$ & $0.9-2.8$ \\
\hline $\begin{array}{l}V_{200, \text { normal tissue }} \\
{\left[\mathrm{cm}^{3}\right]}\end{array}$ & $1.0 \pm 0.6$ & $0.3-1.7$ & $0.9 \pm 0.6$ & $0.2-1.5$ & $0.8 \pm 0.6$ & $0-1.3$ & $0.6 \pm 0.4^{*}$ & $0-1.2$ & $0.7 \pm 0.6^{*}$ & $0-1.4$ \\
\hline \multicolumn{11}{|l|}{ CTV } \\
\hline $\mathrm{V}_{100}[\%]$ & $98.6 \pm 0.8$ & $97.5-99.2$ & $97.7 \pm 1.6$ & $95.3-99.1$ & $96.7 \pm 2.6$ & $92.1-98.8$ & $94.6 \pm 4.0$ & 89.4-98.2 & $92.2 \pm 5.7$ & 82.1-96.4 \\
\hline $\mathrm{V}_{150}[\%]$ & $18.5 \pm 7.8$ & $12-30.1$ & $22.4 \pm 7.0$ & $16.8-33.4$ & $24.0 \pm 7.6$ & $18.8-36$ & $26.6 \pm 6.4$ & $20.5-37.3$ & $28.0 \pm 6.4$ & $22.7-34.9$ \\
\hline $\mathrm{V}_{200}[\%]$ & $4.9 \pm 2.4$ & 2.9-9.5 & $6.4 \pm 2.4$ & $4.5-10.9$ & $7.3 \pm 2.5$ & $5.5-12$ & $9.1 \pm 2.6$ & $6.8-13.4$ & $10.6 \pm 2.2$ & $8.2-13.5$ \\
\hline $\mathrm{D}_{90}[\%]$ & $107.3 \pm 2.8$ & 104.5-109.6 & $108.3 \pm 2.5$ & $104.7-110.7$ & $107.2 \pm 3.6$ & $101.8-110.8$ & $105.6 \pm 4.8$ & $99.5-109.3$ & $103.1 \pm 7.2$ & $92.5-109$ \\
\hline $\mathrm{D}_{100}[\%]$ & $89.3 \pm 4.6$ & 83.1-93.4 & $81.7 \pm 10.2$ & $66.8-91.8$ & $76.5 \pm 13.4$ & $60.7-90.6$ & $68.8 \pm 14.6$ & $52.6-81.6$ & $63.9 \pm 14.1$ & $49.0-75.2$ \\
\hline $\mathrm{D}_{98}[\%]$ & $101.3 \pm 1.9$ & 98.9-103.3 & $99.2 \pm 4.2$ & $94.3-102.9$ & $96.4 \pm 6.8$ & $87.5-102.3$ & $91.1 \pm 9.6$ & $80.9-100.3$ & $85.9 \pm 10.8$ & $75-95.5$ \\
\hline \multicolumn{11}{|l|}{ Urethra } \\
\hline $\mathrm{D}_{\max }[\%]$ & $109.7 \pm 1.2$ & $108.5-110.5$ & $110.2 \pm 0.7$ & $109.2-110.8$ & $110 \pm 1.1$ & 108.3-110.8 & $109.9 \pm 0.8$ & 108.6-110.6 & $110.0 \pm 0.9$ & 109.2-111.5 \\
\hline $\mathrm{D}_{10}[\%]$ & $106.9 \pm 2.0$ & $104.6-108.8$ & $107.3 \pm 1.8$ & 105-108.7 & $106.8 \pm 2.4$ & $104.2-108.9$ & $106.8 \pm 1.9$ & 104.6-108.7 & $106.7 \pm 2.2$ & 103.9-108.6 \\
\hline \multicolumn{11}{|l|}{ Rectal mucosa } \\
\hline $\mathrm{D}_{10}[\%]$ & $57.3 \pm 12.0$ & $41.6-64$ & $57.0 \pm 12.2$ & $40.2-65.7$ & $56.0 \pm 12.4$ & $39-66.2$ & $55.5 \pm 13.4$ & $37.3-63.8$ & $54.8 \pm 13.8$ & $37.2-63.8$ \\
\hline COIN & $0.65 \pm 0.08$ & $0.55-0.73$ & $0.68 \pm 0.08$ & $0.61-0.76$ & $0.71 \pm 0.08$ & $0.64-0.79$ & $0.72 \pm 0.08^{*}$ & $0.66-0.79$ & $0.71 \pm 0.08^{*}$ & $0.61-0.79$ \\
\hline
\end{tabular}

[19] found in a phantom study a geometrical uncertainty for a stainless needle, of 1-3 mm depending on different US-transducers and different US-modes (transversal and sagittal). Milickovic et al. [16] investigated for a similar treatment technique the effect of patient movement and anatomy change on needle and organ dislocation during treatment, and found under these conditions a geometrical shift less than $1 \mathrm{~mm}$. They also pointed out the importance of a good QA to be able to keep the geometrical uncertainties as low as possible by keeping the planning geometry fixed during the treatment procedure.

In this study (Analysis 2) we demonstrated that with the geometrical uncertainty of approximately $2 \mathrm{~mm}$ and with dwell activation $2 \mathrm{~mm}$ outside the CTV, none of the patients reached or exceeded the dose constraints. In order to fulfil the dose constraints for $95 \%$ of the patients, source dwell activation needs to be $5 \mathrm{~mm}$ outside the CTV as a dosimetric margin. Even if this treatment technique has very small geometric uncertainties of around $2 \mathrm{~mm}$ or less, there is a need for a margin on the CTV to guarantee that the dose constraints are met. If a geometrical margin is chosen, there is a need to define a PTV (planning target volume) from the CTV. The results shown in this study are of course dependent on the chosen dose constraints. Changing the dose constraints for a lower $\mathrm{V}_{100, \mathrm{CTV}}$, high- er $D_{\text {max, urethra, }}$ and $D_{10}$ rectal mucosa would probably decrease the dosimetric margin. In a similar simulation study, IKK Kolkman-Deurloo et al. [20] showed that for a treatment technique with multiple fractions per implant, a $3 \mathrm{~mm}$ displacement implied that none of the patients reached the dose constraint $V_{100} \geq 93 \%$. Applying the results in this study based on geometrical uncertainties and a treatment technique with a single fraction per implant, to the same dose constraint used [20] would imply that the dosimetric margin would be in the order of $1-2 \mathrm{~mm}$ and still the $V_{100}$ $\geq 93 \%$ would be achieved. Clinical outcome from other studies with different dose constraints will hopefully give an answer as to the optimal levels of the dose constraints.

There is only a small increase in the COIN-index when going from dwell positioning of $5 \mathrm{~mm}$ to $0 \mathrm{~mm}$ from the CTV surface. This is probably due to the decrease of the coverage $\left(\mathrm{V}_{100}\right)$ of CTV when the irradiated normal tissue outside the CTV is decreasing. As could be seen in Table 1, $\mathrm{D}_{90}$ is not decreasing for the simulation of dwell positioning down to $2 \mathrm{~mm}$, it is actually increasing when going from $5 \mathrm{~mm}(107.3 \%)$ to $3 \mathrm{~mm}(108.3 \%)$ with $p<0.001$. At our hospital we are not using $\mathrm{D}_{90}$ as a dose constraint, since it is regarded as too insensitive compared to $V_{100}$ when analysing dose distributions. 


\section{Conclusions}

It is possible to reduce the treated normal tissue volume by reducing the dwell activation to $3 \mathrm{~mm}$ from the surface and still maintain the dose constraints. However, in combination with the estimated geometrical uncertainties, a dwell activation $5 \mathrm{~mm}$ from the surface is needed to maintain an accurate dose distribution with a $V_{100, \text { CTV }}$ $\geq 97 \%$. This might be seen as a dosimetric margin which could be converted to a geometrical margin and would be evaluated in future work.

\section{Disclosure}

Authors report no conflict of interest.

\section{References}

1. Kovacs G, Galalae R, Loch T et al. Prostate preservation by combined external beam and HDR brachytherapy in nodal negative prostate cancer. Strahlenther Onkol 1999; 175 Suppl 2: 87-88.

2. Edgren M, Ekelund AM, Albertsson P et al. High dose-rate brachytherapy of prostate cancer utilising Iridium-192 after-loading technique: technical and methodological aspects. Int J Oncol 2006; 29: 1517-1524.

3. Borghede G, Hedelin H, Holmang S et al. Combined treatment with temporary short-term high dose rate iridium-192 brachytherapy and external beam radiotherapy for irradiation of localized prostatic carcinoma. Radiother Oncol 1997; 44: 237-244.

4. Hoskin PJ, Motohashi K, Bownes P et al. High dose rate brachytherapy in combination with external beam radiotherapy in the radical treatment of prostate cancer: initial results of a randomised phase three trial. Radiother Oncol 2007; 84: 114-120.

5. Skowronek J. Brachytherapy in the therapy of prostate cancer - an interesting choice. Contemp Oncol (Pozn) 2013; 17: 407-412.

6. Martinez AA, Pataki I, Edmundson G et al. Phase II prospective study of the use of conformal high-dose-rate brachytherapy as monotherapy for the treatment of favorable stage prostate cancer: a feasibility report. Int J Radiat Oncol Biol Phys 2001; 49: 61-69.

7. Zamboglou N, Tselis N, Baltas D et al. High-dose-rate interstitial brachytherapy as monotherapy for clinically localized prostate cancer: treatment evolution and mature results. Int J Radiat Oncol Biol Phys 2013; 85: 672-678.

8. Yoshioka Y, Suzuki O, Otani Y et al. High-dose-rate brachytherapy as monotherapy for prostate cancer: technique, rationale and perspective. J Contemp Brachytherapy 2014; 6: 91-98.

9. Hoskin P, Rojas A, Ostler P et al. High-dose-rate brachytherapy alone given as two or one fraction to patients for locally advanced prostate cancer: acute toxicity. Radiother Oncol 2014; 110: 268-271.

10. Edmundson GK, Rizzo NR, Teahan M et al. Concurrent treatment planning for outpatient high dose rate prostate template implants. Int J Radiat Oncol Biol Phys 1993; 27: 1215-1223.

11. Edmundson GK, Yan D, Martinez AA. Intraoperative optimization of needle placement and dwell times for conformal prostate brachytherapy. Int J Radiat Oncol Biol Phys 1995; 33: 1257-1263.

12. Lahanas M, Baltas D, Zamboglou N. Anatomy-based threedimensional dose optimization in brachytherapy using multiobjective genetic algorithms. Med Phys 1999; 26: 1904-1918.

13. Pierquin B, Dutreix A, Paine $\mathrm{CH}$ et al. The Paris system in interstitial radiation therapy. Acta Radiol Oncol Radiat Phys Biol 1978; 17: 33-48.
14. Giannouli S, Baltas D, Milickovic N et al. Autoactivation of source dwell positions for HDR brachytherapy treatment planning. Med Phys 2000; 27: 2517-2520.

15. Baltas D, Kolotas C, Geramani K et al. A conformal index (COIN) to evaluate implant quality and dose specification in brachytherapy. Int J Radiat Oncol Biol Phys 1998; 40: 515-524.

16. Milickovic N, Mavroidis P, Tselis N et al. 4D analysis of influence of patient movement and anatomy alteration on the quality of 3D U/S-based prostate HDR brachytherapy treatment delivery. Med Phys 2011; 38: 4982-4993.

17. Palmer A, Bradley D, Nisbet A. Physics-aspects of dose accuracy in high dose rate (HDR) brachytherapy: source dosimetry, treatment planning, equipment performance and in vivo verification techniques. J Contemp Brachytherapy 2012; 4 : 81-91.

18. Kirisits C, Rivard MJ, Baltas D et al. Review of clinical brachytherapy uncertainties: analysis guidelines of GEC-ESTRO and the AAPM. Radiother Oncol 2014; 110: 199-212.

19. Siebert FA, Hirt M, Niehoff $P$ et al. Imaging of implant needles for real-time HDR-brachytherapy prostate treatment using biplane ultrasound transducers. Med Phys 2009; 36: 34063412 .

20. Kolkman-Deurloo IK, Roos MA, Aluwini S. HDR monotherapy for prostate cancer: a simulation study to determine the effect of catheter displacement on target coverage and normal tissue irradiation. Radiother Oncol 2011; 98: 192-197. 